\title{
Space-VLBI with RadioAstron: new correlator capabilities at MPIfR
}

\author{
G. Bruni ${ }^{1 *}$, J. M. Anderson ${ }^{1,2}$, W. Alef ${ }^{1}$, A. Lobanov ${ }^{1,3}$, J. A. Zensus ${ }^{1}$ \\ ${ }^{1}$ Max-Planck-Institut für Radioastronomie, Auf dem Hügel 69, 53121 Bonn, Germany \\ ${ }^{2}$ Deutsches GeoForschungsZentrum GFZ, Telegrafenberg A6, 14473 Potsdam, Germany \\ ${ }^{3}$ Institut für Experimentalphysik, Universität Hamburg, Luruper Chaussee 149, 22761 Hamburg, \\ Germany \\ E-mail: bruni@mpifr-bonn.mpg.de
}

DiFX is a correlator for VLBI data based on the FX architecture (first Fourier transform and then cross-multiply). DiFX is a free licensed software written in $\mathrm{C}++$, developed and maintened by an international group of programmers.

A new DiFX version ( $d r a$ ) has been developed at Max-Planck-Institut für Radioastronomie (MPIfR), in order to manage the correlation of a space-based antenna with ground stations.

The dra version is running on the High Performance Computer cluster (HPC) in Bonn, and it is used for the data processing of the three AGN imaging RadioAstron Key Science Projects ongoing, based at the MPIfR.

12th European VLBI Network Symposium and Users Meeting,

7-10 October 2014

Cagliari, Italy

\footnotetext{
*Speaker.
} 


\section{The RadioAstron mission}

RadioAstron is an international space VLBI (SVLBI) mission led by the Astro Space Center (ASC, Moscow) and utilizing a 10-meter space radio telescope (SRT) on board of the Russian satellite Spektr-R which was launched in July 2011 [2]. Since beginning of 2012, it is performing interferometric observations with ground-based telescopes from all over the world. Key science projects as well as PI-driven projects have been observed until now during Announcement of Opportunity 1 (AO-1, July 2013 - July 2014), and AO-2 (July 2014 - July 2015). The MPIfR plays a leading role in three KSPs aimed to study structure, evolution, and polarisation properties of extragalactic jets, taking advantage of the extreme angular resolution offered by RadioAstron. Since summer of 2013, the Bonn correlator is processing data from these projects: during AO-1, 13 experiments were observed for the three KSPs.

The correlation of the collected data is carried out at the High Performance Computer (HPC) cluster, based at MPIfR. It features 480 cores, 13 RAIDs ( $650 \mathrm{~Tb}$ of storage capability), $20 \mathrm{Gbps}$ infiniband interconnection, and 15 Mark5 units for diskpacks playback. The HPC cluster is currently used also for correlation of mm-VLBI, and geodetic projects.

\section{The dra version of DiFX}

As a contribution to the RadioAstron mission, the MPIfR devoted efforts to enhance the DiFX correlator [1] to enable space-VLBI (SVLBI) correlation including the RadioAstron telescope. Although the RadioAstron mission continues to develop its own software correlator in the Astro Space Center (ASC) Moscow, the mission desired having an independent correlator available to be able to check the ASC correlator results. Furthermore, enhancing the DiFX correlator for SVLBI enables the MPIfR to perform correlation of RadioAstron experiments for MPIfR science projects as a contribution to the RadioAstron mission, and since DiFX is widely used by the VLBI community, RadioAstron correlation could in principle be performed by any other DiFX correlator group around the world.

During initial data format testing in 2012, D. Graham (MPIfR) wrote software to convert the RadioAstron raw-voltage data format (RDF) to the Mark5B file format based on reverse engineering the sample data obtained from the RadioAstron mission. This enables the RadioAstron data to be converted into a format supported by DiFX. J. M. Anderson later modified this conversion software to correct for changes made to the format since 2010 and to incorporate documentation on the data format obtained in 2012.

Initial efforts to modify the DiFX correlator software itself were started in 2011. The most significant part of this effort involved modifying the delay model server (Calc, from the Calc/Solve package $^{1}$ ) to be able to calculate delay information for telescopes with arbitrary coordinates and velocities (that is, not fixed on the ground), and changes to the DiFX metadata system to deal with the changing position and velocity of the spacecraft as a function of time. The delay model was also modified to correct for (general) relativistic effects as the highly elliptical orbit of the spacecraft results in large changes in velocity and gravitational potential compared to the terrestrial frame. Because of hardware issues in the equipment used for receiving and recording the RadioAstron

\footnotetext{
${ }^{1}$ http://gemini.gsfc.nasa.gov/solve/
} 


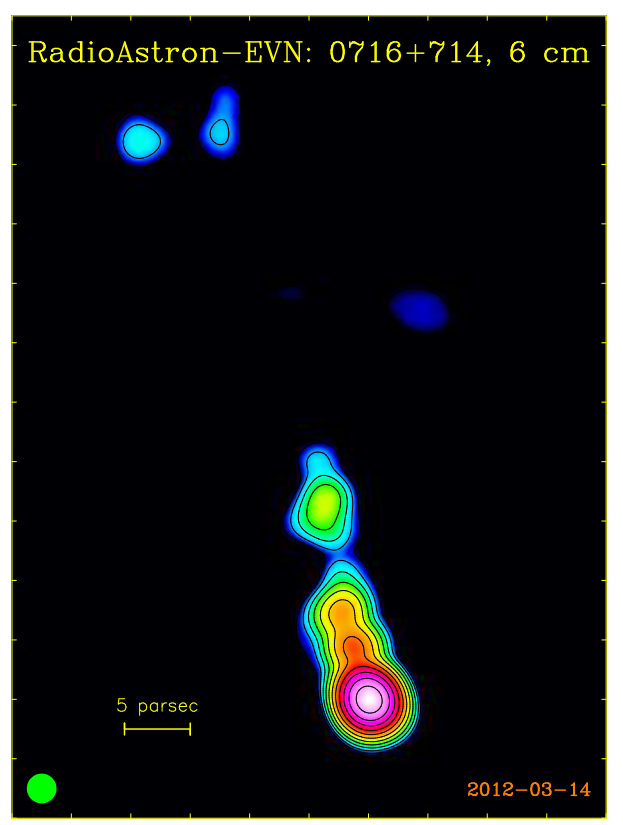

Figure 1: Image of the active galaxy $0716+714$ at $4.9 \mathrm{GHz}$ made from data produced by the $d r a$-DiFX correlation of EVN project EK032C observed on 2012 March 14th, including baselines to RadioAstron [2]. $0716+714$ is a BL Lac-type object that was selected for the initial RadioAstron imaging commissioning observations because of its compact core, high declination allowing continuous ground observation, and sky location matching RadioAstron's observing constraints for the time of year.

VLBI datastream on the ground station(s), and because of inherent limitations in the data timestamp information provided by RadioAstron, the timestamp information for the RadioAstron data are actually set by the tracking station clock at the moment it begins recording individual VLBI scans. Modifications to DiFX therefore also involved changes to the metadata handler in DiFX and to the delay model server to incorporate not only the relative time of arrival of the astronomical signal at the spacecraft, but also to calculate the delay for the transmission of the signal from the spacecraft to the tracking station.

First fringes obtained with the developed DiFX version, named $d r a$, were obtained on 2012 June 14 using observations of BL Lac on the RadioAstron-Effelsberg baseline, and resulting in a clear peak at one location. Approximately same solution in the delay-rate space was found by the ASC correlator for the same dataset. Following the initial fringe result and continued development and commissioning of the $d r a$-DiFX correlator, it was used to correlate the first imaging experiment involving RadioAstron. Figure 1 shows the initial imaging result obtained by processing the EK032C experiment [2], using AIPS and DIFMAP for calibration and imaging, respectively. The output datasets from the DiFX software have also been used to make direct comparisons with the ASC correlator results. Such comparisons identified a problem in the ASC $(u, v, w)$ calculations, which has subsequently been fixed.

$d r a$-DiFX is presently used only at the MPIfR, for correlation of RadioAstron data. The merging with the publicly available dtrunk version of the DiFX correlator is ongoing. In the following, a summary of the additional capabilities and modifications: 
- RDF-Mark5B conversion routine, to read in data from RadioAstron spaceborne antenna

- Enabling delay model server Calc (Calc/Solve Package) to calculate delay information for a spaceborne antenna

- Introducing general relativistic corrections in the delay model

- Changing DiFX metadata system to deal with variable position/velocity of the spaceborne antenna

- Calculating the delay for the transmission of the signal from the spacecraft to the tracking station

- Calculating the equivalent of parallactic angle correction for the spaceborne antenna from the antenna orientation obtained from the telemetry information.

- Wide fringe-search windows can be used, thanks to the customized fringe-fitting software.

Future improvements will include:

- Spacecraft acceleration terms correction

- Inclusion of the on-board maser information from telemetry data

- Automatic conversion of the native RDF data format to Mark5B

\section{References}

[1] Deller, A., Brisken, W. F., Phillips, C. J. et al. 2011, PASP, 123, 275

[2] Kardashev, N. S., Khartov, V. V., Abramov, V. V. et al. 2013, Astronomy Reports, Vol. 57, Issue 3, 153 